\title{
An Attitude Based Cooperative Negotiation Model in a Hostile Multi-agent World
}

\author{
Madhu Goyal \\ University of Technology \\ PO BOX 123 \\ Broadway NSW 2007 \\ Australia
}

\begin{abstract}
In multi-agent setting agent teams often encounter conflicts in agents' plans and actions. This paper presents a cooperative negotiation model $(\mathrm{ABCON})$ that allows agents in a team to appropriately negotiate various options in a hostile and dynamic fire world. It shows that negotiations explore the attitudes and behaviors that help agents to manage conflict constructively. It says that cooperative negotiation is guided by the agents' dynamic assessment of alternative actions given the different scenario conditions. The application and implementation of this model to a virtual fire- fighting domain has revealed a promising prospect in negotiating conflicts and solving them.
\end{abstract}

\section{Introduction}

The negotiation theory has received a great deal of attention from the multi-agent system community [5][8][11][12]. Negotiation is the process of searching for an agreement that satisfies various parties. It is an ongoing process that occurs against a backdrop of continuing relationships and events. Negotiations are like a chess match during conflicts in cooperative environment; have a strategy; anticipate how the other will respond; how important is the issue and situation for you. Thus a negotiation usually involves a number of steps including the exchange of proposals and counter proposals. During the negotiation process an agreement may be reached through the facilitation of the achievement of cooperation among entities such as individuals, groups or teams.

This paper presents a model for negotiation with specific application to a fire world. We say that based on the beliefs that arise in the process of conflict resolution in the hostile dynamic environment, we formulate specific attitudes about the strategies we will use in the negotiation. These may be quite general (e.g. Plan to use a cooperative approach) or quite specific (eg. Use a specific negotiating tactic.). Finally attitudes are translated into behavior. These behaviors in turn elicit some response from the other agent and the process goes on. Our objective is the development of a simulation environment of a real world situation, in which conflicts can be resolved amicably through negotiations. We aim to design cooperative agents, which can survive in a hostile dynamic world and solve problems with other similar agents inhabiting the same world. These agents will be capable of interacting with each other both individually and collectively. In addition, they will be capable of deriving plans and negotiating over the resources in order to achieve a common goal. Negotiation mechanism depends heavily on the specific characteristics of the scenarios under consideration. In hostile dynamic domain the passage of time during the negotiation is the most important consideration. Also negotiations as a way of reaching mutual benefit are a costly and time-consuming process and, consequently, it may increase the overhead of cooperation and coordination. In the presence of time constraints, planning and negotiation time [16] 
should be taken into consideration. Kraus et al. [9] analyzed a number of problems related to resource allocation and task distribution among self-motivated, rational and autonomous agents while involved in multi-agent negotiation under time constraints. Their approach crucially tackle the issue of instantaneous outcomes: given that the negotiation cost is taken into consideration, the negotiation should end as quickly as possible, possibly in the first round. The attitude based negotiation mechanism besides taking care of negotiation time also takes into consideration different alternative options and commitments of collaborating agents.

\section{Fire World Domain}

The team problem solving ideas are implemented and tested on a simulation of a fire world FFTEAMS using a virtual research campus. The idea of simulated fire world was first given in Phoenix [2], which is a real time, adaptive planner that manages forest fires in simulated environment. The virtual campus is implemented using C++ on Windows 2000 platform, where more than 40 agents share the world via network. FFTEAMS is a dynamic, distributed, interactive, simulated fire environment where agents are working together to solve problems, for example, rescuing victims and extinguishing fire. The fire world FFTEAMS that we have considered in this paper consists of a large number of objects (of the order of hundreds) and several agents. It consists of several buildings, an open ground area, walkways, a car park, and campus gates. Objects in the fire world include walls, buildings, furniture, open areas and LPG gas tanks. There are two types of agents: victim agents and fire fighting agents. A part of the fire world is shown in figure 1. My world is different from others' (like Air Combat [13] and RoboCup [7]) in respect that problems posed to the agents and the changes in the environment are not only caused by the actions of other agents but also by the changes the objects themselves undergo in the world (caused by the fire). In a world such as this, no agent can have full knowledge of the whole world. Humans and animals in the fire world are modeled as autonomous and heterogeneous agents. While the animals run away from fire instinctively, the fire fighters can tackle and extinguish fire and the victims escape from fire in an intelligent fashion. An agent responds to fire at different levels. At the lower level, the agent burns like any object, such as chair. At the higher level, the agent reacts to fire by quickly performing actions, generating goals and achieving goals through plan execution. This world contains all the significant features of a dynamic environment and thus serves as a suitable domain for our team agents. Agents operating in the domain face a high level of uncertainty caused by the fire. Agents in the fire domain do not face the real time constraints as in other domains, where certain tasks have to be finished within the certain time. However, because of the hostile nature of the fire, there is strong motivation for an agent to complete a given goal as soon as possible. The communication and co-operation are the essentials to accomplish fire-fighting tasks and to achieve proper control of the hostile situation.

The team behaviors play an integral role in a Fire World. There are three main objectives for intelligent agents in the world during the event of fire: self-survival, saving objects including lives of animals and other agents and put-off fire. Because of the hostile settings of the domain, there exist a lot of challenging situations where agents do the team activities. Whenever there is fire, the basic team behavior is exhibited by the fire fighters. The fire fighters perform all the tasks necessary to control an emergency scene. The problem solving activities of the fire fighters are putting out fire, rescuing victims and saving property. Apart from these primary activities there are a number of sub tasks e.g. run towards the exit, move the objects out of the room, remove obstacles, and to prevent the spread of fire. In order to carry out the teamwork successfully the team of fire fighters should carry out number of procedures. There are number of preconditions of team behavior in the fire world. First, the firefighters should work in pairs whenever they are in a hazardous or potentially hazardous location on the emergency scene. Firefighters working alone may over exert themselves or be unable to help themselves when trapped. Second, a fire 
attack must be coordinated to be successful. The fire fighters must perform the desired activity at the time when the officer wants them to perform. Depending on the conditions at the fire scene, the fire fighters may choose to perform immediate rescue or to protect exposures rather than attacking the fire. Coordination between team members performing different functions is crucial. For example, when carrying hose-lines the team of fire fighters requires an explicit coordination technique.

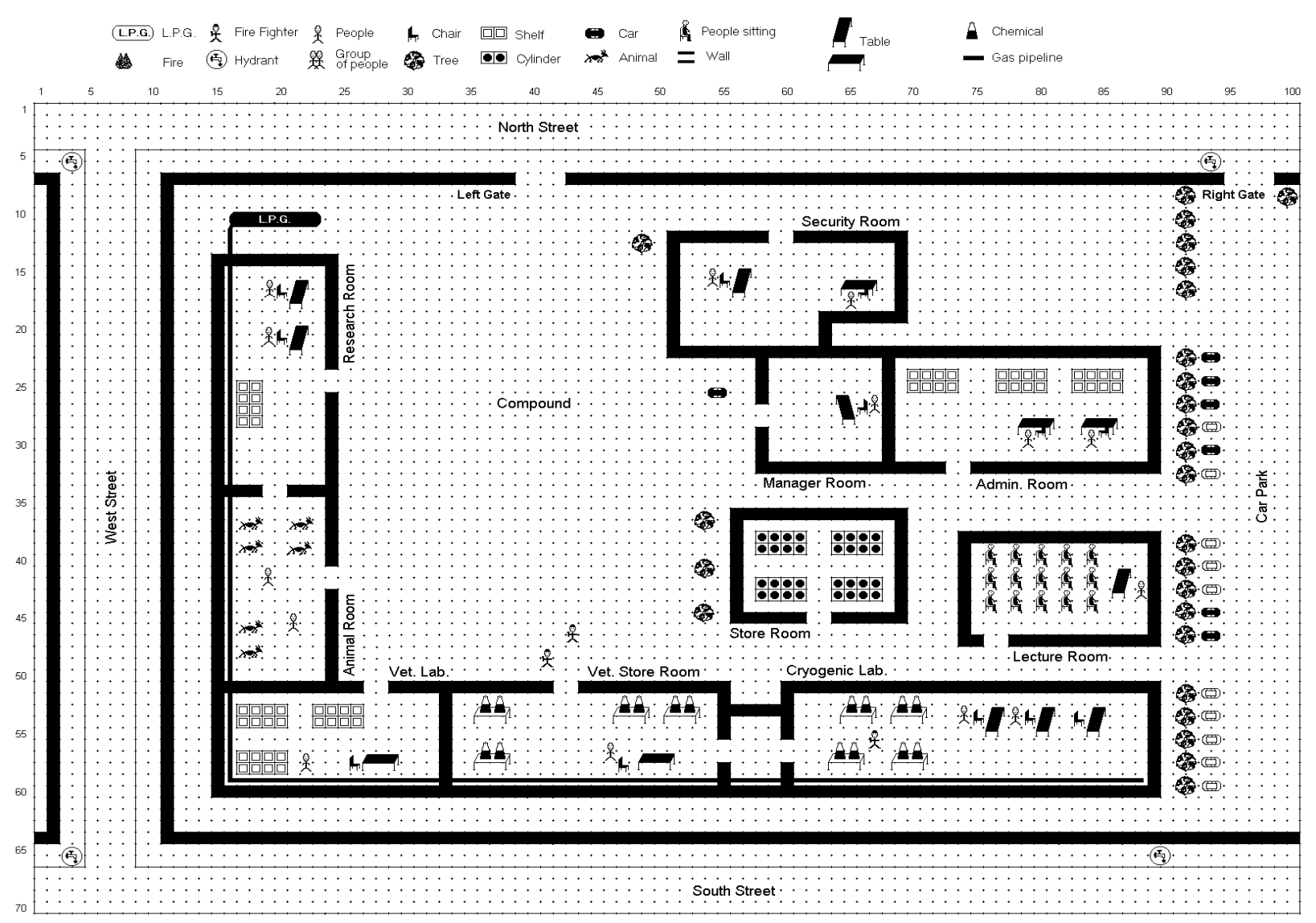

Figure 1: A Fire World

\section{A Framework for Cooperative Negotiation}

The negotiation process facilitates the achievement of cooperation among different agents. The cooperation among agents succeeds only when participating agents are enthusiastically unified in pursuit of a common objective rather than individual agendas. The negotiation is a higher-level discourse function involving distinct acts to reach a group consensus. We claim that cooperation among agents is achieved only if the agents have a collective attitude towards cooperative goal as well as towards cooperative plan. There are two views toward negotiation: focus on a particular task, or focus on a decision that may involve consideration of multiple alternative tasks. In this paper, a key aspect of collaboration is negotiating about alternative ways to achieve goals. To support such negotiation, we have extended our representation so that task models support reasoning about alternative, mutually exclusive courses of action (recipes) for achieving tasks, and we have added mechanisms for evaluating the relative strengths and weaknesses of different alternatives. We use heuristic approach to resolve our conflicts in a team. Such methods acknowledge that there is decision-making involved with computation 
and so seek to search the negotiation space in a non-exhaustive fashion. The key advantages of the heuristic approach are that it is based on realistic assumptions and can use different alternate options.

\subsection{Definition of Attitude}

Attitude is a learned predisposition to respond in a consistently favourable or unfavourable manner with respect to a given object [4]. But we define attitude as a predisposition to respond consistently in favourable or unfavourable manner with respect to a given object. In other words, the attitude is a preparation in advance of the actual response, constitutes an important determinant of the ensuing behaviour. However this definition seems too abstract for computational purposes. In AI, the fundamental notions to generate the desirable behaviours of the agents often include goals, beliefs, intentions, and commitments. Goal is a subset of states, and belief is a proposition that is held as true by an agent. Bratman [1] addresses the problem of defining the nature of intentions. Crucial to his argument is the subtle distinction between doing something intentionally and intending to do something. The former case might be phrased as deliberately doing an action, while intending to do something means one may not be performing the action in order to achieve it. Cohen and Levesque [3], on the other hand, developed a logic in which intention is defined. They define the notion of individual commitment as persistent goal, and an intention is defined to be a commitment to act in a certain mental state of believing throughout what he is doing. Thus to provide a definition of attitude that is concrete enough for computational purposes, we model attitude using goals, beliefs, intentions and commitments. From the Fishbein's [4] definition it is clear that when an attitude is adopted, an agent has to exhibit an appropriate behaviour (predisposition means behave in a particular way). The exhibited behaviour is based on a number of factors. The most important factor is goal or several goals associated with the object. During problem solving, an agent in order to exhibit behaviour may have to select from one or several goals depending on the nature of the dynamic world.

In a dynamic multiagent world, the behaviour is also based on appropriate commitment of the agent to all unexpected situations in the world including state changes, failures, and other agents' mental and physical behaviours. An agent intending to achieve a goal must first commit itself to the goal by assigning the necessary resources, and then carry out the commitment when the appropriate opportune comes. Second, if the agent is committed to executing its action, it needs to know how weak or strong the commitment is. If the commitment is week, the agent may not want to expend too much of its resources in achieving the execution. The agent thus needs to know the degree of its commitment towards the action. This degree of commitment quantifies the agent's attitude towards the action execution. For example, if the agent considers the action execution to be higher importance (an attitude towards the action), then it may choose to execute the action with greater degree of commitment; otherwise, the agent may drop the action even when it had failed at the first time. Thus, in our formulation, an agent when it performs an activity, since the activity is more likely that it will not succeed in a dynamic world, agents will adopt a definite attitude towards every activity while performing that activity. The adopted attitude will guide the agent in responding to failure situations.

\subsection{Attitude Based Team Model}

We claim that team activity is achieved only if the agents have team as a collective abstract attitude. From collective attitudes, agents derive individual attitudes that are then used to guide their behaviors to achieve the team activity. We consider two agent team in this paper i.e. $A_{1}$ and $A_{2}$. So the collective attitude of the agent $A_{1}$ and $A_{2}$ towards the collection team is represented as $\operatorname{Team}_{A I A 2}\left(A_{1}, A_{2}\right)$. But from $A_{1}$ 's viewpoint, team is an attitude that it is holding towards the collection $\left(A_{1}, A_{2}\right)$ and can be denoted as $\operatorname{Team}_{A l}\left(\mathrm{~A}_{1}, \mathrm{~A}_{2}\right)$. Similarly from $\mathrm{A}_{2}$ 's viewpoint, its attitude can be denoted as $\operatorname{Team}_{A 2}\left(\mathrm{~A}_{1}, \mathrm{~A}_{2}\right)$. But the 
collective attitude $\operatorname{Team}_{A 1}{ }_{A 2}\left(\mathrm{~A}_{1}, \mathrm{~A}_{2}\right)$ is decomposed into the individual attitudes only when both the agents mutually believe that they are in the team. In order to establish mutual belief between the agents, the agents have to commit to the team activity by saying so. Besides mutual belief, we model a team using a set of four attributes i.e. team definition $\left(\mathrm{DEF}^{\mathrm{T}}\right)$, team methods $\left(\mathrm{M}^{\mathrm{T}}\right)$, team rule base $\left(\mathrm{R}^{\mathrm{T}}\right)$ and team responsibility $\left(\operatorname{RESP}^{\mathrm{T}}\right)$. Thus $\operatorname{Team}_{A i}\left(\mathrm{~A}_{1}, \mathrm{~A}_{2}\right)$, the abstract attitude of a team agent can be divided into two components (i) attitude towards team definition $\left(\mathrm{DEF}^{\mathrm{T}}\right)$, team methods $\left(\mathrm{M}^{\mathrm{T}}\right)$, team rule base $\left(\mathrm{R}^{\mathrm{T}}\right)$, mutual belief $\left(\mathrm{MB}^{\mathrm{T}}\right)$ and team responsibility $\left(\mathrm{RESP}^{\mathrm{T}}\right)$ (ii) behaviors towards the components of team structure i.e. towards that team definition $\left(\mathrm{DEF}^{\mathrm{T}}\right)$, team methods $\left(\mathrm{M}^{\mathrm{T}}\right)$, team rule base $\left(\mathrm{R}^{\mathrm{T}}\right)$, mutual belief $\left(\mathrm{MB}^{\mathrm{T}}\right)$ and team responsibility $\left(\mathrm{RESP}^{\mathrm{T}}\right)$. The overall attitude towards the team attributes is further divided into the attitudes towards each component using the same attitude decomposition technique described above.

\subsubsection{Team Definition $\left(D_{E} F^{T}\right)$}

The team definition attribute $\left(\mathrm{DEF}^{\mathrm{T}}\right)$ specifies the definition of the team. Each agent uses this definition to verify whether the team exists at any point of time. The team definition can be implemented in a number of ways. However, the most important point in the implementation of team definition is that each agent in the team is individually aware of the team, and "consciously" supports the team as an abstract entity by performing appropriate team definition activities. In a hostile dynamic environment, the world state and the behaviours of the agents are unpredictable. In order to keep pace with the changing world an agent has to verify, whether the agents are still in the team or not. In our implementation of team definition, each agent sends a query to other agent asking whether it is in team or not. If the answer is positive the team exists, otherwise it does not. Accordingly, every agent in a team will follow set of rules defined by team eg. to check whether two agents $A_{1}$ and $A_{2}$ are in a team or not, we have a pair of question answer session between two agents. To establish this session $A_{1}$ first asks $A_{2}$ : Are you in the team? If $\mathrm{A}_{2}$ is in team it will reply with the answer: Yes, $\mathrm{I}$ am in the team. If $\mathrm{A}_{1}$ does not receive an answer within a reasonable time, or the received reply is "No", it will unform the team. Thus the function of team definition attribute $\left(\mathrm{DEF}^{\mathrm{T}}\right)$ is to check whether agents are in the team or not. In our model, this team definition attribute $\left(\mathrm{DEF}^{\mathrm{T}}\right)$ can be invoked periodically or whenever there is a change in the situation.

\subsubsection{Team Rule Base $\left(\mathbf{R}^{\mathrm{T}}\right)$}

The team rule base $\left(\mathrm{R}^{\mathrm{T}}\right)$ contains a set of rules, which generates various team behaviours in different conditions. The team rule base $\left(\mathrm{R}^{\mathrm{T}}\right)$ determines what different team goals or sub-goals are to be generated under what conditions. These behaviours of the team agents are represented in the form of condition/action pairs where conditions are logical expressions over the inputs and actions lead to behaviours when executed. To clarify the concept of the team rule base, part of the rule base or behaviours of a fire fighting team is shown in Fig. 2.

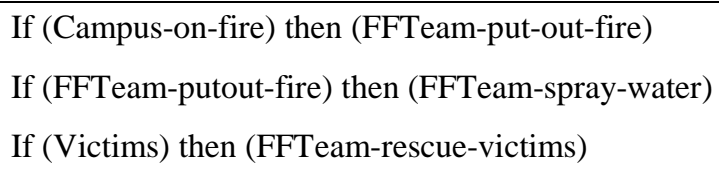


Figure 2: Fire-Fighting Team's Rule Base

\subsubsection{Team Methods $\left(\mathbf{M}^{\mathrm{T}}\right)$}

In our team model, the team method $\left(\mathrm{M}^{\mathrm{T}}\right)$ attribute specifies the problem solving methodology for achieving the team goals. A team problem can be solved in a number of different ways. When the team agents examine the problem besetting the team, each team agent invokes the team methods $\left(\mathrm{M}^{\mathrm{T}}\right)$ attribute to get information about the methods to solve the problem. The team methods $\left(\mathrm{M}^{\mathrm{T}}\right)$ attribute provides every agent the knowledge of the particular method it is using and details of using this method in order to achieve a particular team task or goal. For example, in the fire world, the fire fighting agents should know how to put out the fire in various situations.

\subsubsection{Mutual Beliefs $\left(\mathrm{MB}^{\mathrm{T}}\right)$}

The mutual belief attribute $\left(\mathrm{MB}^{\mathrm{T}}\right)$ provides every team agent with mutual belief in order to achieve the team goal. As discussed above, the teamwork by a team does not merely consist of simultaneous and coordinated individual actions, but team agents should mutually believe the commitment to the team goal. In order to establish this mutual belief, each team agent tries to be responsive and committed to the actions of the other team agent.

\subsubsection{Responsibility $\left(\right.$ RESP $\left.^{\mathrm{T}}\right)$}

Responsibility [6] defines conditions, which must be satisfied before joint action can start and specifies a code of conduct for agents once problem solving has commenced. According to it for collaborative problem solving, joint persistent goal [3] is not sufficient. The responsibility attribute of the team gives consideration to how goals can be achieved (plan sates) or how participants should behave or have solution commitment when engaged in collaborative problem solving. However, we define responsibility as an attitude of team agents, which defines its commitments towards team goal and team plan execution. We say that, when agents are in team, they should have appropriate attitudes towards individuals, groups, and society, as well as the team. Thus when a team of agents undertake a problem, responsibility as an attitude prescribes each member to perform its part precisely till the problem is solved and the team is dissolved. However, while doing the team activity in the dynamic environment, it is inadequate for the agent to commit to the team activity only. The team agents are autonomous participants that perform specialised functions within a social setting. Thus besides team tasks concerning with the operation of the entire team, we need to the agents to perform activities specific to non-global subtasks. Striving towards a team goal, however, does not imply that every action by every agent need to be team action, since conflict at a local level may occur without compromising the global goals. The conflicts in a team can be due to autonomy of agents, group-work and social obligations. In this context, social conventions and individual behaviours provide general guidelines which agents can follow. By adopting a convention, every agent knows what is expected of it, and of every other agent, as a part of collective working towards the goal, and implicitly knows that every other agent has a similar set of expectations. Thus besides doing team activity, the team agent also has to do social actions, group work and individual actions. Our solution (responsibility as an attitude) provides a means of maximizing the predictability of the agents and the team as a whole. The idea is powerful in that it provides ideas about cooperative scheduling and planning as well as collision/conflict avoidance. Its richness presents numerous possibilities for studying different patterns of individual, group and social behaviour. Thus the responsibility attribute $\left(\mathrm{RESP}^{\mathrm{T}}\right)$ specifies the responsibility of each agent towards the team. Without the 
responsibility attribute the agents in a team can not work out the preconditions necessary for each team action and how to act in a social or group setting. From the multiagent perspective, an agent's responsibility can be divided into the following major subcategories: (1) individual responsibility $\left(\operatorname{RESP}^{\mathrm{Ti}}\right)$ (2) group responsibility $\left(\mathrm{RESP}^{\mathrm{Tg}}\right)$ (3) social responsibility $\left(\mathrm{RESP}^{\mathrm{Ts}}\right)$ (4) team responsibility $\left(\mathrm{RESP}^{\mathrm{Tt}}\right)$. Thus the individual commitments, social commitments and group commitments parameterise our idea of responsibility. All of these agent's responsibilities provide the necessary background knowledge to guide agent's actions and allow them to exhibit appropriate behaviours in a complex, dynamic multiagent world. The benefit of this multi-level structured view of agent's responsibility is that it provides a basis for the agents to work out their activities and conflicts in such a way that they don't violate the social and group norms.

\subsection{Attitude Based Cooperative Negotiations (ABCON)}

Figure 3 presents the overall $\mathrm{ABCON}$ negotiation process. The ABCON has three steps i.e. Opening State, Negotiation State, Closing State.

1. Opening State:

(a) Team ${ }_{\mathrm{A} 1 \mathrm{~A} 2}\left(\mathrm{~A}_{1}, \mathrm{~A}_{2}\right)$ detects a conflict.

(b) Team believes that it can resolve the conflict

(c) Else team withdraw the negotiation process.

2. Negotiation state:

(a) For time less than deadline $t_{\max }$, any member in the team may generate proposal to resolve conflict.

(b) Team's responsibility attribute evaluate the proposal.

(c) If no conflict found within deadline, accept the proposal and go to step 3

(d) Else if proposal found conflict, continue negotiation through counter-offer.

3. Closing state:

(a) If suggested proposal accepted terminate the negotiation process.

(b) Else if conflict found unachievable, irrelevant or the deadline to resolve the conflict is passed, then terminate the negotiation state.

Figure 3: Stages in $A B C O N$

We say that, when agents are in team, they should also have appropriate attitudes towards individuals, groups, and society, as well as the team. Thus when a team of agents undertake a problem, an attitude prescribes each member to perform its part precisely till the problem is solved and the team is dissolved. However, while doing the team activity in the dynamic environment, it is inadequate for the agent to commit to the team activity only. The team agents are autonomous participants that perform specialised functions within a social setting. Thus besides team tasks concerning with the operation of the entire team, we need that the agents to perform activities specific to non-global subtasks. Striving towards a team goal, however, does not imply that every action by every agent need to be team action, since conflict at a local level may occur without compromising the global goals. The conflicts in a team can be due to autonomy of agents, group-work and social obligations. In this context, social conventions and individual behaviors provide general guidelines which agents can follow. By adopting a convention, every agent knows what is expected of it, and of every other agent, as a part of collective working towards the goal, and implicitly knows that every other agent has a similar set of expectations. Thus besides doing team activity, the team agent also has to do social actions, group work and individual actions. If the team attitude of agents is high (high degree of commitment), the agents in team follow the team plan or go according to the team agenda. If the attitude of agents in team is medium, agents in team may or may not follow the team guidelines. Instead they may follow group, society or individual plans 
depending on the attitude of agents against each entity. If the attitude of agents in team is low, agents in team will not at all follow the team plan. A heuristic model to evaluate the proposal given to one team agent to another team agent is given in figure 2 .

Another important factor effecting the negotiations in a hostile dynamic world is a deadline $t_{\max }$ set by the team to conclude its negotiation for the problem. In case of conflicts in team, the agents follow an alternating sequential protocol, which has five different stages i.e. offer, counter-offer, accept, decline and withdraw. An offer is a proposal made by the agent to the other, counter-offer is a proposal from an agent in response to proposal it received, accept is to accept a proposed offer, decline is to reject the previously accepted offer and withdraw is to terminate the negotiation.

High Team Attitude Medium Team Attitude Low Team Attitude High Group Attitude
Medium Group Attitude
Low Group Attitude

High Social Attitude Medium Social Attitude Low Social Attitude

High Individual Attitude Medium Individual Attitude Low Individual Attitude accept team offer consider (may or may not accept) team offer decline team offer

accept group counter-offer consider (may or may not accept) group counter-offer decline team offer

accept social counter-offer consider (may or may not accept) social counter-offer decline social offer

\section{Evaluation}

accept individual counter-offer

consider (may or may not accept) individual counter-offer decline individual offer

Figure 4: A Heuristic model for Proposal Evaluation

The key evaluation criterion for the overall performance of the team in any environment is that agents must successfully accomplish their tasks within their given environments, both efficiently and accurately. We have done several experiments for fire fighting in FFWorld domain to verify our ideas about the cooperative negotiation model. To assess the utility of our model a series of experiments were undertaken. The motivation behind doing these experiments is to determine the advantages and disadvantages of our attitude based cooperative negotiation model. The agents in a team react to the changes in the world states by generating and achieving new goals. Meanwhile, old goals and plans are constantly being monitored and re-structured if necessary. The attitudes are mainly concerned with how to re-organise plans and goals due to situational changes. Whenever there is a new goal because of the changes in the world, one or more attitudes are usually created along with the team goal. The experiments have concentrated on evaluating the performance of attitude based team in case of unexpected events. 


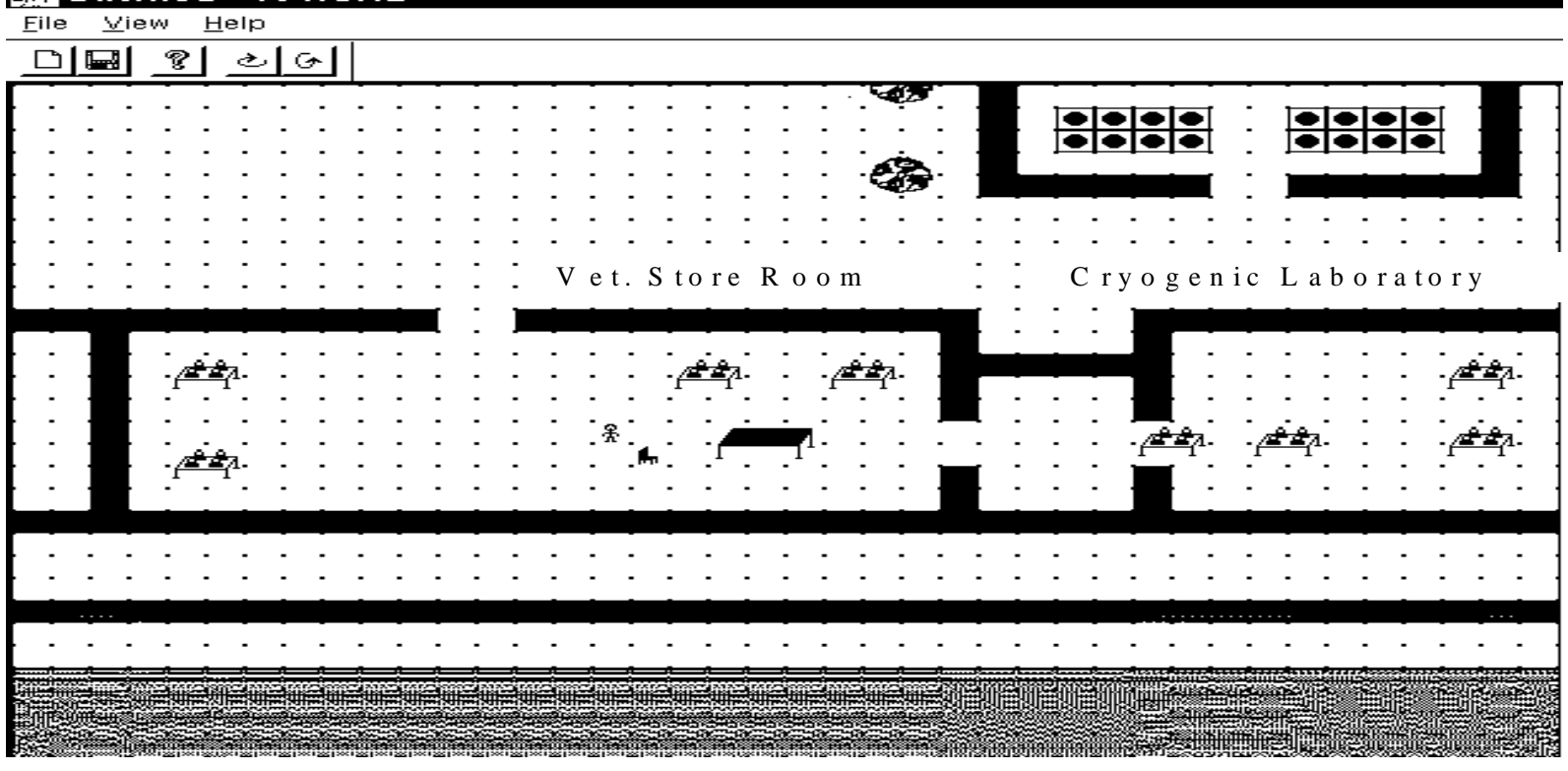

Figure 5: A Section of FFWorld Showing the Entrance of the Cryogenic Laboratory Blocked by a Table

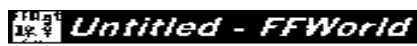

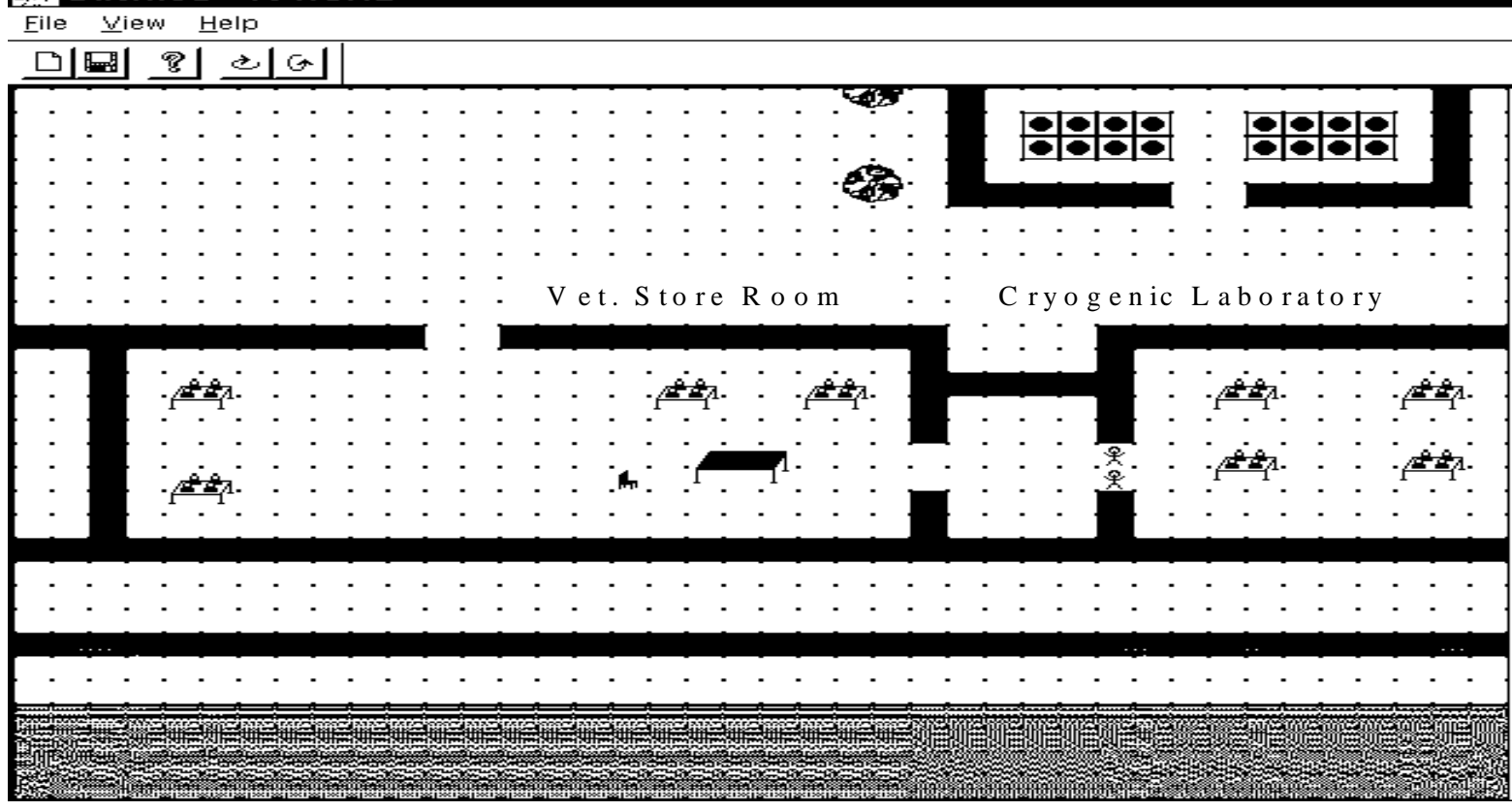

Figure 6: A Section of FFWorld Showing the Entrance of the Cryogenic Laboratory Blocked by two agents

When a problem occurs, the attitude model stipulates a new set of behaviours for the team agents. The team agents with attitude respond to changes in the world by adopting a set of attitudes towards these changes. We carried out different types of experiments to investigate the problem solving behaviour of the team agents during team action and to analyse their coherence in hostile dynamic environments. 
These sets of experiments demonstrate the significance of employing attitudes when team agents have to deal with individual, group, social and team goals in a changing world. A problem for a multiagent system is how it can maintain global coherence(how well a system behaves as a unit) without explicit global control. In this case, the agents must be able on their own to determine goals they share with other agents, determine common tasks, avoid unnecessary conflicts, and pool knowledge. Since the overall goal of fire-fighting teams is to put out fire, it is also interesting and informative to note that how the team performs against a wide range of unseen adversaries and how it can operate in a dynamic, hostile environment.

In this experiment, we have tried to find the probability of success in case of three types of teams (i) both agents in the team having high attitude towards team- $\mathrm{T}_{\text {high }}$ (ii) both agents in the team having medium attitude $-\mathrm{T}_{\text {medium }}$ (iii) agents in the team having low attitude $-\mathrm{T}_{\text {low. }}$. The performance of three types of team agents is measured in terms of the rate of change of world and time taken to complete the task. The high attitude based team $\mathrm{T}_{\text {high }}$ performs better than the low attitude based team $\mathrm{T}_{\text {low }}$, because attitude based agents can easily detect problems and can develop commitments accordingly. The team $\mathrm{T}_{\text {high }}$ performs better than the team $\mathrm{T}_{\text {medium }}$ and $\mathrm{T}_{\text {low }}$, because the agent with high attitude can better guide the team to react to unforeseen adversaries. In case of low attitude based team $\mathrm{T}_{\text {low }}$, the agents would not be able to respond to the changes in the world. They will not be able to detect the safe and unsafe conditions of the world and not able to resolve conflicts when they have to coordinate with other agents to use some

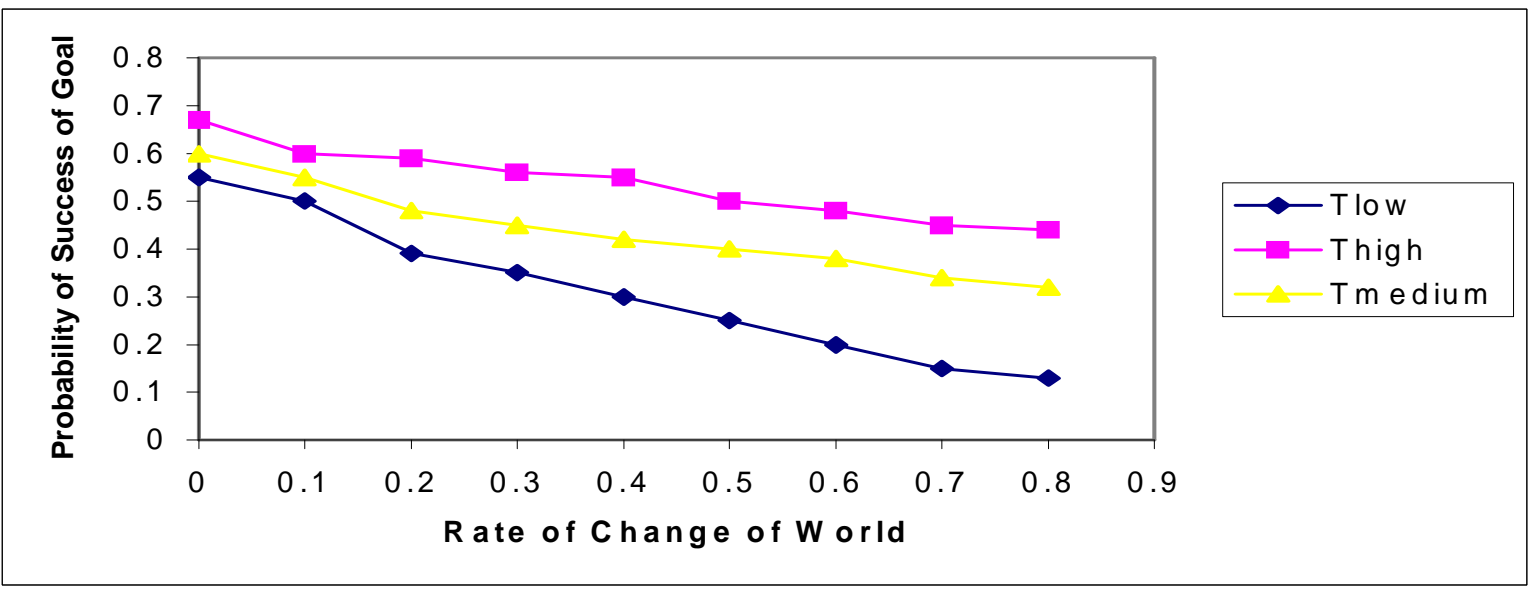

common resources.

Figure 7: Probability of Success of Team Goal for the Teams $T_{\text {high }}, T_{\text {medium }}$ and $T_{\text {low. }}$.

Figure 7 shows the graph of the experiment measuring the probability of success of goal when the state of the world is changing rapidly. The x-axis of the graph shows the rate of change of the world, while the $y$-axis represents the probability of success of goal. We measure the rate of change of world in terms of the pace with which the fire spreads out. We observe more severe the situation is (from small fire to large fire), the more efforts the team spends in achieving the same goal (put out the fire). In the meantime, the chances of plan failures increase as the fire world becomes more hostile. This is understandable since medium and large fire creates more difficulties, and poses more challenges for team's planning and plans execution. We note that when the fire is small, the team plan seems to do well, because there are less chances of plan failure. So the number of rules fired is also less. We also found that probability of success of goal in case of high attitude based teams are more, while in case of low attitude based team is less. Similarly, the less attitude based agents will not be able to handle the blockage of the entrance of the room by the agent or by an object (table or chair). There are greater chances of removing the blockage at the entrance in case of attitude based agents. In our experiment, while going to the Cryogenic Lab. the 
team of fire-fighters find that entrance to the lab. is blocked by the table (figure 5). The team agents responsibility attribute generates social and group attitudes like help and full-coordination. The team goal generator will insert a new goal $\mathrm{G}_{\text {move-the- table }}$ into the time line. With the insertion of this new goal, there are greater chances of the team agents achieving the main goal i.e. put out fire. Because the new attitudes adopted will help the team agents to move the table to a safe place. Similarly, if one agent is blocking the exit (figure 6), the another agent have to wait for some time. The team agents' attitude generator will generate social attitudes like wait. Then the team goal generator will insert a new goal $\left(\mathrm{G}_{\mathrm{wait}}\right)$ into the time line depicting the team's social behaviour. These results show that pure interest in team is not a good basis for cooperation. Participation in team problem solving requires some element of compromise i.e. team interests need to be tempered with the consideration for the individuals, groups and society. The cooperative negotiation model helps agents to resolve conflicts due to individual goals, group goals and social goals and generate appropriate solutions to the local problems of the team agents with the help of various attitudes. Whenever a problem occurs in the fire world, the team attitude model stipulates that a new course of action should be devised. The unexpected events in the fire world cause the team plan violation and distract the agents from its intended commitments. In order to simulate the team plan violation, varying numbers of unexpected tasks were assigned to the team during the lifetime of the team action. The agent acting as an individual, group and social agent could solve all these additional tasks. We observe that the chances of plan failures increase, as the world becomes more hostile. This is understandable since large fire creates more difficulties, and poses more challenges for team's planning and plan execution. We note that when the fire is small, the team plan seems to do well, because there are less chances of plan failure.

\section{Related Work}

Early work on negotiations was based on game theory, such as [11] [16]. In another approach, PERSUADER [12] enriched the negotiation model by integrating AI planning, Case Based Reasoning and other decision theoretical techniques. The system involves three agents: 1) a union, 2) a company, and 3) a mediator and it operates in the domain of labour negotiation. It models iterative exchange of proposals and counter proposals for the parties to reach agreement. Agreement can be reached through persuasive argumentation. Later, Bazaar [15] a sequential decision-making negotiation model that is capable of learning is developed. Within the negotiation framework, learning is modelled as a Bayesian belief update process. Experimental results show that building learning capability into agents' decision making helps agents to form more accurate model of the opponents and results in better performance and less expensive process. Kraus [9] focuses on negotiation under time constraints. Using a distributed mechanism, agents conduct negotiation and can reach agreements without delays. The study comes to a conclusion that delaying agreements causes inefficiency in the negotiation.

The model of Alternating Offers [10] is applied to the negotiation protocol and it operates on problems with both complete and incomplete information. Experimental results show that strategic negotiation performs better than the static allocation policy currently used for data allocation for servers in distributed systems. In Collaborative Negotiation Systems based on Argumentation (CONSA) [14], agents negotiate by providing arguments in support of their proposals to one another. The key concept of CONSA is to cast conflict resolution as an explicit common team goal, therefore if a team member privately discovers an event that renders the current team conflict irrelevant, it will make this fact mutual to its team members. My work is similar to alternating offers and CONSA, but my negotiation model provides the more probability of collaborative successful negotiations in a hostile dynamic world.

\section{Conclusions}


This paper has developed a heuristic cooperative model for managing negotiations in a hostile dynamic world. Negotiation is guided by the agent's dynamic assessment of alternative actions given the current scenario conditions, with the aim of facilitating the agents in a team to complete their tasks as quickly as possible. Our solution provides a means of maximizing the predictability of the agents and the team as a whole. The idea is powerful in that it provides ideas about cooperative scheduling and planning as well as collision/conflict avoidance. Its richness presents numerous possibilities for studying different patterns of individual, group and social behaviour. The team model presented in this paper refines and formalises the notion of team as viewed as a team member. It grounds the physical and mental activities of a set of agents as a cohesive "team" in the attitudes of its individual members and towards the team attributes. The team model presented in this paper has the following features: Team is a collective attitude (albeit implicit) of the abstract team agents. At the same time, team is also an individual, but explicit attitude of the participating agents (thus team is modelled at two levels). In attitude based team model, a group of agents may perform an activity, which is based on mutual beliefs and joint intentions, but unless they are intended as a team activity, they cannot be viewed as a team activity. In a hostile and dynamic world, it is necessary that individual agents have their own attitudes towards such important attributes as mutual belief, responsibility, team definition, team rule base and team methods. The different attitudes of agents towards these attributes provide agents with greater flexibility and greater chance of successful negotiations.

\section{References}

[1] M.E. Bratman. Intentions, Plans and Practical Reason. Harvard University Press, Cambridge, MA, 1987.

[2] P.R. Cohen, M.L.Greenberg, D.M. Hart, and A.E. Howe. Trial by fire: Understanding the design requirements for agents in complex environments. AI Magazine, 10(3), 1989.

[3] P.R.Cohen and H.J.Levesque. Teamwork. Special Issue on Cognitive Science and Artificial Intelligence, 25(4), 1991.

[4] M. Fishbein and I. Ajzen. Belief, Attitude, Intention and Behaviour: An Introduction to theory and research. Reading, MA,USA:Addison-Wesley, 1975.

[5] N.R.Jennings, P.Faratin, A.R.Lomuscio, S.Parsons, C.Sierra, and M.Wooldrige. Automated negotiation: prospects, methods and challenges. Group Decision and Negotiation, 10(2): 199-215,2001.

[6] N.R.Jennings. Controlling Cooperative problem solving in Industrial Multi-agent Systems using Joint Intentions. Journal of Artificial Intelligence 74 (2), 1995.

[7] H.Kitano, M. Tambe, P. Stone, M. Veloso, S.Coradeschi, E. Osawa, H. Matsubara, I. Noda, and M.

Asada. The RoboCup Synthetic Agent Challenge,97, International Joint Conference on Artificial Intelligence (IJCAI97).

[8] S.Kraus. Strategic Negotiation in Multiagent Environments. The MIT press, 2001.

[9] S.Kraus, J.Wilkenfeld, and G.Zlotkin. Multiagent negotiation under time constraints. Artificial Intelligence, 2(75):297-345,October 1995. 
[10] M.J.Osborne and A.Rubinstein. Bargaining and Markets. Academic Press Inc., San Diego, California, 1990.

[11] J.S.Rosenchein and G.Zlotkin. Rules of Encounter: Designing Conventions for automated negotiation among Computers. The MIT Press, Cambridge, MA, 1994.

[12] K.Sycara. Problem restructuring in negotiation. Management Science, 37(10), 1991.

[13] Milind Tambe. Towards Flexible Teamwork. In Journal of Artificial Intelligence Research 7(1997), pp 83-124.

[14] Milind Tambe and Hyuckchul Jung. The Benefits of Arguing in a Team. AI-Magazine Vol 20, November, 1999.

[15] D. Zeng and K. Sycara. Benefits of Learning in Negotiation. In Proceedings of the National Conference on Artificial Intelligence, 36-41, Menlo Park, California.

[16] Gilad Zlotkin and Jefferey S. Rosenchein. Negotiation and task sharing among autonomous agents in cooperative domains. In Proceedings of the Eleventh International Conference Joint Conference on Artificial Intelligence, pages 912-917, August 1989. 